\title{
AVALIAÇÃO DO PERFIL DE DISSOLUÇÃO DE MEDICAMENTOS UTILIZANDO-SE ESPECTROSCOPIA ELETRÔNICA MULTIVARIADA
}

\author{
Juliana dos Santos, Gilcélia A. Cordeiro, Noemi Nagata e Patricio Peralta-Zamora* \\ Departamento de Química, Universidade Federal do Paraná, CP 19081, 81531-990 Curitiba - PR, Brasil \\ Letícia Norma Carpentieri Rodrigues \\ Departamento de Ciências Exatas e da Terra, Universidade Federal de São Paulo, 09972-270 Diadema - SP, Brasil
}

Recebido em 21/1/11; aceito em 3/5/11; publicado na web em 8/7/11

\begin{abstract}
EVALUATION OF THE DISOLUTION PROFILE OF DRUGS BY MULTIVARIATE ELECTRONIC SPECTROSCOPY. In this work the evaluation of the dissolution profile of captopril-hydrochlorothiazide and zidovudine-lamivudine associations were carried out by multivariate spectroscopic method. The models were developed by partial least square regression from 20 synthetic mixtures using mean-centered spectral data. The external validation was accomplished with 5 synthetic mixtures shown mean prevision error of about $1 \%$. Good agreement was observed in the analyses of commercial drugs (content uniformity and dissolution profile), considering the results obtained by the standard chromatographic method, with prevision error lower than $10 \%$.
\end{abstract}

Keywords: captopril-hydrochlorotiazide; zidovudine-lamivudine; multivariate spectroscopy.

\section{INTRODUÇÃO}

No meio industrial, a utilização de ferramentas de análise em rotinas de controle de qualidade se mostra essencial, principalmente para garantir a qualidade dos produtos comercializados. Na indústria farmacêutica, principalmente na área de produção de medicamentos, este tipo de controle se apresenta ainda mais relevante, em razão da necessidade de se garantir, além da uniformidade de conteúdo, características como segurança e ação esperada.

Dentro deste contexto, os testes de dissolução se mostram particularmente importantes, na medida em que permitem, por medições in vitro, avaliar a disponibilidade do fármaco in vivo. De forma simplificada, a dissolução pode ser entendida como a liberação de um fármaco da sua forma farmacêutica, tornando-se disponível para absorção pelo organismo. Desta forma, o ensaio de dissolução corresponde a um teste físico, no qual o fármaco passa para a forma solúvel a partir da forma farmacêutica sólida intacta ou dos fragmentos formados durante o ensaio. ${ }^{1}$

Em geral, considera-se que a dissolução do produto no organismo é o fator limitante para a disponibilidade fisiológica do fármaco. Portanto, medidas da taxa de dissolução, ou de um parâmetro relacionado, oferecem indicação significativa da disponibilidade fisiológica. Caso exista uma correlação entre dissolução e algum parâmetro de biodisponibilidade, o simples procedimento de monitorar os perfis de dissolução deve permitir a predição da disponibilidade in vivo. ${ }^{2}$

O elevado custo dos estudos in vivo e a necessidade de técnicos qualificados e de seres humanos para investigação ressaltam a importância dos testes de dissolução como medição indireta da biodisponibilidade do fármaco, especialmente em avaliações preliminares da formulação.

Normalmente, as técnicas analíticas utilizadas na avaliação dos ensaios de dissolução são as mesmas utilizadas no controle de qualidade das matérias-primas e dos produtos acabados, com marcada predominância de métodos cromatográficos, principalmente em fase líquida. ${ }^{2-4}$ Por sua vez, métodos fundamentados em espectroscopia ele-

*e-mail: zamora@.ufpr.br trônica são relatados com menor frequência, usualmente envolvendo a avaliação de medicamentos que contêm apenas um fármaco. ${ }^{5,6}$ Para misturas mais complexas, os problemas de interferência espectral costumam inviabilizar o uso de sistemas convencionais de calibração, o que praticamente exige o uso de sistemas de calibração fundamentados em espectrofotometria derivativa ${ }^{7}$ ou calibração multivariada. ${ }^{8}$

Além de uma interessante revisão relacionada com o uso de ferramentas de calibração multivariada em análises biomédicas, ${ }^{9}$ importantes aplicações na área farmacêutica têm sido recentemente publicadas em revistas nacionais. ${ }^{10-12}$ Entretanto, poucos trabalhos são encontrados na literatura sobre o desenvolvimento de testes de dissolução de medicamentos por espectroscopia eletrônica associada a ferramentas de calibração multivariada. Dentro deste contexto, destaque pode ser dado aos bons resultados relatados por Dinç e colaboradores, que avaliaram o perfil de dissolução de medicamentos contendo paracetamol, propifenazona e cafeína. ${ }^{13}$

Neste trabalho, a regressão de mínimos quadrados parciais (PLS) está sendo proposta para viabilizar a determinação espectroscópica simultânea das associações captopril-hidroclorotiazida e lamivudinazidovudina, importantes medicamentos utilizados no tratamento da hipertensão arterial e da síndrome de imunodeficiência adquirida (AIDS), de maneira a permitir a avaliação do perfil de dissolução de medicamentos.

Normalmente, a determinação simultânea destas espécies em amostras de medicamentos é realizada por cromatografia líquida, principalmente em função da existência de métodos padrão referenciados na Farmacopeia Norte Americana. ${ }^{14}$ Até onde se pode investigar, não há relatos do uso de ferramentas multivariadas para a determinação espectroscópica simultânea de captopril/hidroclorotiazida e lamivudina/zidovudina.

\section{PARTE EXPERIMENTAL}

Amostras e padrões

Captopril (CAP, 1-[(2S)-3-mercapto-2-metilpropionil]-L-prolina) 
e hidroclorotiazida (HIDRO, 1,1 dióxido de 6-cloro-3,4-di-hidro-2H1,2,4-benzotiadiazina-7-sulfonamida) foram gentilmente fornecidos pelos laboratórios Pharma Nostra e Henrifarma, respectivamente, com pureza de $99 \%$. Padrões foram preparados por dissolução dos fármacos em solução aquosa de etanol $(10 \% \mathrm{v} / \mathrm{v})$.

Zidovudina (AZT, 3'-azido-3'-deoxitimidina) e lamivudina (3TC, 2'-deoxi-3'-tiacitidina) foram gentilmente fornecidos pelo Laboratório Cristália Produtos Químicos e Farmacêuticos Ltda., com pureza de $99 \%$. Padrões foram preparados por dissolução direta dos fármacos em água deionizada.

As amostras de medicamentos foram adquiridas no comércio local, correspondendo a medicamentos de referência, genérico e similar.

Outros reagentes (ácido, bases, solventes e sais) foram de grau analítico PA.

\section{Desenvolvimento de modelos multivariados de calibração}

Para o desenvolvimento de modelos multivariados foram produzidas 25 misturas, contendo 20,0 a $30,0 \mathrm{mg} \mathrm{L}^{-1}$ de CAP e 10,0 a 15,0 $\mathrm{mg} \mathrm{L}^{-1}$ de HIDRO (ou 19,00 a 29,00 $\mathrm{mg} \mathrm{L}^{-1}$ de AZT e 10,00 a $14,00 \mathrm{mg} \mathrm{L}^{-1}$ de $3 \mathrm{TC}$ ). Esta faixa foi definida levando-se em consideração o teor nominal dos fármacos em medicamentos comercialmente disponíveis, a variação máxima permitida pela legislação (normalmente $\pm 20 \%$ ) e o coeficiente de absortividade apresentado por cada fármaco.

Os modelos foram desenvolvidos a partir de 20 misturas, enquanto que as 5 restantes (selecionadas em torno do ponto central) foram reservadas para a fase de validação externa. Para melhorar a eficiência dos modelos multivariados, foram utilizados procedimentos de transformação e pré-processamento de dados, dentre os quais se destacam: dados centrados na média, dados alisados, dados alisados e derivados e, dados autoescalados.

Os espectros de absorção UV-Vis foram registrados entre 190 e $400 \mathrm{~nm}$ (captopril-hidroclorotiazida) e entre 190 e $300 \mathrm{~nm}$ (zidovunina-lamivudina), utilizando-se espectrofotômetro Shimadzu (2410 PC) e cubetas de quartzo de $1 \mathrm{~cm}$ de caminho óptico.

Para desenvolvimento dos modelos de calibração multivariada (PLSR) foi utilizado o pacote de programas do PLS-toolbox 3.0 (Eigenvector), o qual opera em ambiente Matlab 6.5 (MathWork). As matrizes foram construídas utilizando o software Origin $6.1^{\circledR}$ (OriginLab).

\section{Análise de medicamentos comerciais}

O modelo de calibração multivariada de melhor desempenho (avaliado pelo conjunto de validação externa) foi utilizado na determinação quantitativa das espécies de interesse em amostras do medicamento de referência, genérico e similar, as quais contêm $50 \mathrm{mg}$ de captopril e $25 \mathrm{mg}$ de hidroclorotiazida (ou $300 \mathrm{mg}$ de zidovudina e $150 \mathrm{mg}$ de lamivuina), por comprimido.

A massa média dos comprimidos foi determinada utilizandose 20 amostras. Posteriormente, estes 20 comprimidos foram triturados e homogeneizados. Finalmente, uma massa equivalente a 1 comprimido foi tomada e dissolvida em 1000,00 mL de água deionizada (zidovudina e lamivudina) ou solução aquosa de etanol ( $10 \%$ v/v, captopril-hidroclorotiazida). Após filtração em membrana de acetato de celulose $(0,45 \mu \mathrm{m})$, diluições convenientes foram realizadas com água deionizada e solução aquosa de etanol, respectivamente.

$\mathrm{Na}$ análise por cromatografia em fase líquida foi utilizado um procedimento similar, desta vez utilizando-se a fase móvel para dissolução e diluição das amostras.

Todas as análises foram realizadas em triplicata.

\section{Ensaios de dissolução}

Ensaios de dissolução foram realizados de acordo com especificações da ANVISA, ${ }^{15}$ utilizando-se dissolutor Hanson SR6 composto por 6 cubas de $1 \mathrm{~L}$.

Para a associação captopril e hidroclorotiazida foi utilizado o aparato 1 (cesta) e meio constituído por $\mathrm{HCl} \mathrm{0,1} \mathrm{mol} \mathrm{L-1} \mathrm{(900} \mathrm{mL),}$ enquanto que para a associação de zidovudina e lamivudina foi utilizado o aparato 2 (pá) e meio constituído por água deionizada e degaseificada (900 mL). É importante salientar que estas condições configuram um procedimento padrão para a realização dos ensaios, basicamente em função de permitirem que a dissolução dos fármacos ocorra em condições que não acompanhem a saturação do meio, ou seja, de maneira análoga ao verificado no trato gastrointestinal (condição SINK).

Ambos os ensaios foram realizados em quintuplicata, com controle de temperatura $\left(37 \pm 0,5^{\circ} \mathrm{C}\right)$ e agitação de $50 \mathrm{rpm}$, coletando-se alíquotas em tempos de 1, 3, 5, 7, 10, 15, 20 e 30 min. As alíquotas foram imediatamente filtradas em membrana de acetato de celulose $(0,45 \mu \mathrm{m})$, diluídas quando necessário e analisadas por método espectroscópico multivariado e cromatográfico padrão.

\section{Análise cromatográfica}

As análises por cromatografia líquida de alta eficiência foram realizadas de acordo com método padrão da Farmacopeia Norte Americana. ${ }^{14}$

As determinações de captopril e hidroclorotiazida foram realizadas em cromatógrafo Varian 920-LC, equipado com detector ultravioleta $(210 \mathrm{~nm})$ e coluna Microsorb-MV 100/5 C8 ( 250 x 4,6 x 1/4"), utilizando-se fase móvel constituída de água deionizada:metanol:ácido fosfórico (750:250:0,5 v/v). A curva analítica foi preparada a partir de 8 misturas sintéticas, contendo entre 2,00 e $60,00 \mu \mathrm{g} \mathrm{mL}^{-1}$ de captopril e 1,00 e $30,00 \mu \mathrm{g} \mathrm{mL} \mathrm{mL}^{-1} \mathrm{de}$ hidroclorotiazida, dissolvidas diretamente em fase móvel.

As determinações de zidovudina e lamivudina foram realizadas em cromatógrafo Varian Pró-Star, equipado com coluna Chompack C18 (250 x 4,6 x 1/4") e detector UV (265 nm). Como fase móvel foi utilizada uma mistura de acetonitrila:água deionizada:metanol $(70: 440: 490 \mathrm{v} / \mathrm{v})$. A curva analítica foi preparada a partir de 8 misturas sintéticas, contendo entre 10,00 e $60,00 \mu \mathrm{g} \mathrm{mL}^{-1}$ de zidovudina e 5,00 e $30,00 \mu \mathrm{g} \mathrm{mL}^{-1}$ de lamivudina, dissolvidas diretamente em fase móvel.

\section{RESULTADOS E DISCUSSÃO}

\section{Associação captopril-hidroclorotiazida}

A partir dos espectros das substâncias puras (Figura 1), é possível verificar que o captopril apresenta apenas uma banda de absorção centrada em 199,5 nm, a qual é intensamente interferida pelo sinal da hidroclorotiazida. Para este segundo fármaco observam-se duas bandas de absorção, uma centrada em 225,5 nm e outra em 271,0 $\mathrm{nm}$, sendo que esta última se apresenta livre de interferências espectrais nas concentrações utilizadas no estudo. Em primeira análise, a quantificação convencional de hidroclorotiazida parece possível, principalmente utilizando-se o sinal registrado em 271,0 nm. Para captopril, a necessidade de métodos diferenciados é evidente, em razão da forte interferência espectral observada. Adicionalmente, é importante salientar que existe uma grande semelhança entre os espectros das misturas sintéticas e das amostras de medicamento (Figura 1), o que, pelo menos em princípio, sugere pouca interferência por parte dos excipientes que fazem parte da formulação (especificidade). 


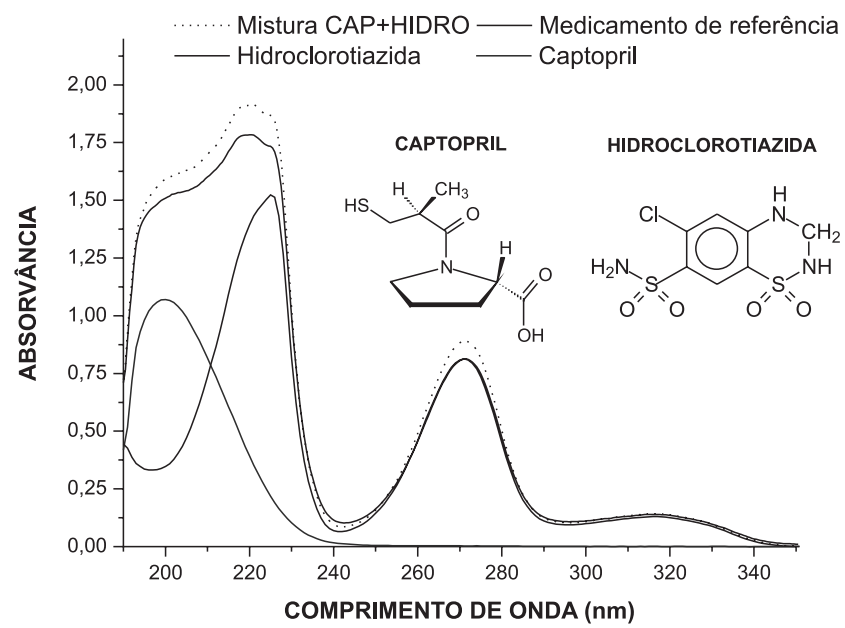

Figura 1. Espectro eletrônico de soluções aquosas de captopril (25,0 $\left.\mathrm{mg} \mathrm{L}^{-1}\right)$, hidroclorotiazida $\left(12,5 \mathrm{mg} \mathrm{L}^{-1}\right)$, mistura sintética e medicamento de referência

Inicialmente, os modelos multivariados foram desenvolvidos utilizando-se espectros centrados na média, pré-processamento que costuma ser utilizado para corrigir problemas associados ao deslocamento da linha de base. Para definir o melhor número de variáveis latentes (VL), foi utilizado o critério da minimização do valor de RMSECV (raiz quadrada do somatório dos erros de previsão ao quadrado) no processo de validação cruzada (sistema leave one $o u t$ ). De acordo com a evolução deste parâmetro (resultados não apresentados), os erros de previsão são minimizados pelo uso de duas VL, as quais permitem representar $99,75 \%$ da variância dos dados espectrais e $98,79 \%$ da variância dos dados de concentração. A inclusão de um maior número de VL contribuiu muito pouco com a variância total explicada, ao mesmo tempo em que provocou um significativo aumento no erro de previsão de captopril.

Para verificar a presença de anomalias no conjunto de calibração foram utilizados os critérios de resíduos de Student e "Leverage". Os resíduos de Student indicam se as amostras estão incluídas na distribuição normal, considerando-se um intervalo de confiança de $95 \%$, enquanto que o "Leverage" corresponde a um parâmetro que representa a influência de um espectro em particular, podendo ser entendido como a distância entre cada espectro e o centroide do conjunto de espectros. Utilizando-se estes critérios (resultados não apresentados) não foram observadas anomalias no conjunto de calibração.

Objetivando-se o aprimoramento dos modelos de calibração, foram realizados estudos com diversos tipos de pré-processamento de sinais e diferentes números de VL (2, 3, 5, 7 e $10 \mathrm{VL})$. Os resultados, apresentados na forma de erro médio na etapa de validação externa (Figura 2), indicam que todos os modelos desenvolvidos apresentam capacidade de previsão comparável, fornecendo erros médios inferiores a 2\%. Para aplicação em análises subsequentes foi selecionado o modelo elaborado com espectros centrados na média (pré-processamento 1) e duas variáveis latentes, que proporciona erros médios de previsão inferiores a $1 \%$ para ambos os fármacos.

\section{Validação do método multivariado}

Com o intuito de validar o método multivariado, foram realizados estudos de acordo com os critérios exigidos pela ANVISA, envolvendo a avaliação de parâmetros de repetibilidade, reprodutibilidade e robustez, este último frente a mudanças de $\mathrm{pH}$, temperatura e tempo de leitura. Neste estudo, foi utilizado o modelo multivariado de melhor desempenho, ou seja, aquele desenvolvido com 2 VL e espectros centrados na média.

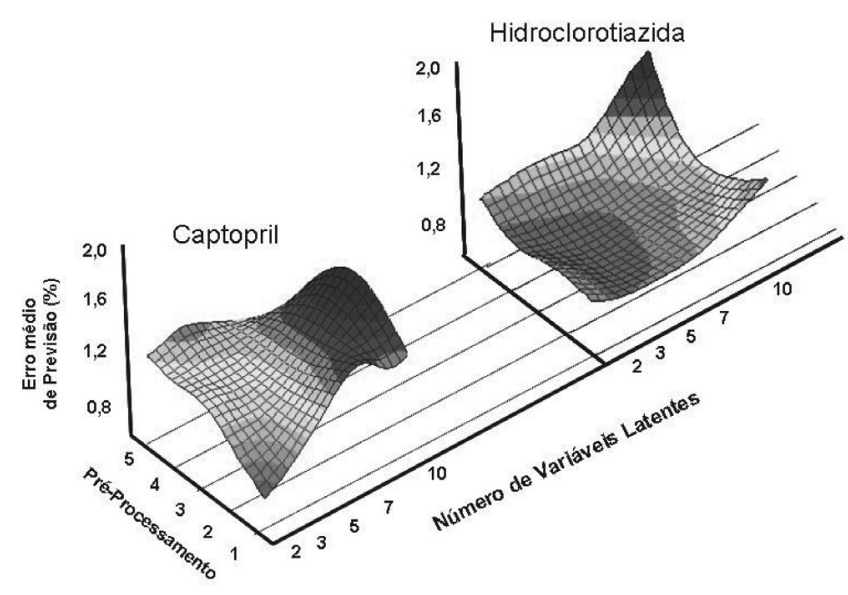

Figura 2. Evolução do erro médio de previsão (captopril-hidroclorotiazida) na fase de validação externa em função do número de variáveis latentes e do tipo de pré-processamento (1: centrado na média, 2: autoescalado, 3: alisado e derivado, 4: alisado, 5: derivado)

O teste de repetibilidade avalia a diferença entre repetições do mesmo ensaio, executado no mesmo laboratório, dentro de um curto período de tempo, com o mesmo analista e a mesma instrumentação. Os resultados desta avaliação (não apresentados) demonstram uma excelente precisão da metodologia analítica em avaliação, com desvios padrão relativos entre 0,15 e $1,31 \%$ e, portanto, dentro dos limites estabelecidos pela legislação vigente $(5 \%)$. O teste de reprodutibilidade é semelhante ao de repetibilidade, com a diferença de que a análise deve ser realizada por outro analista, em outro laboratório e utilizando-se outro equipamento. Os resultados obtidos neste ensaio são apresentados na Tabela 1, demonstrando a obtenção de desvios padrão relativos (DPR) bem inferiores ao valor limite imposto pela norma específica $(5 \%)$.

Adicionalmente, os resultados apresentados na Tabela 1 permitem avaliar a exatidão da metodologia proposta, em função da recuperação das concentrações utilizadas no ensaio. Neste caso, a recuperação alcança valores entre 96,1 e 99,5\%, o que pode ser considerado satisfatório.

A avaliação da robustez deve ser considerada durante a fase de desenvolvimento do método, constatando-se suscetibilidade a variações nas condições analíticas, as quais deverão ser adequadamente controladas ou incluídas como precauções no procedimento. Neste caso, a etapa mais importante na construção do modelo é a obtenção dos espectros das espécies de interesse. Portanto, a principal observação a ser feita está representada pelas condições experimentais que possam alterar a resposta instrumental e, consequentemente, os resultados de previsão.

A influência da temperatura foi avaliada entre 5 e $45^{\circ} \mathrm{C}$, utilizando-se a mistura que melhor representa a composição dos medicamentos em estudo (CAP: 25,00 $\mathrm{mg} \mathrm{L}^{-1}$ e HIDRO: 12,50 mg $\mathrm{L}^{-1}$ ). Em primeira análise, as mudanças de temperatura não provocaram nenhum efeito significativo no perfil espectral das amostras, o que permitiu a obtenção de resultados coerentes em toda a faixa de temperatura estudada, com desvios padrão relativos inferiores a $2 \%$ (Tabela 1).

Resultados bastante similares foram observados com relação ao tempo de leitura, desta vez com desvios padrão relativos ainda menores (Tabela 1).

$\mathrm{Na}$ avaliação do efeito do $\mathrm{pH}$ do meio, foram observadas mudanças espectrais significativas, principalmente envolvendo as bandas centradas em 200 e $225 \mathrm{~nm}$. A referida modificação espectral altera a capacidade de previsão dos modelos multivariados (Tabela 1), 
Tabela 1. Resultados da determinação de captopril e hidroclorotiazida em misturas sintéticas utilizadas para avaliação da reprodutibilidade $(\mathrm{n}=3)$ e robustez

\begin{tabular}{|c|c|c|c|c|}
\hline \multirow{2}{*}{ Concentração } & \multicolumn{2}{|c|}{ Captopril } & \multicolumn{2}{|c|}{ Hidroclorotiazida } \\
\hline & $\begin{array}{c}\text { Média } \\
\left(\mathrm{mg} \mathrm{L}^{-1}\right)\end{array}$ & $\begin{array}{l}\text { DPR } \\
(\%)\end{array}$ & $\begin{array}{l}\text { Média } \\
\left(\mathrm{mg} \mathrm{L}^{-1}\right)\end{array}$ & $\begin{array}{c}\text { DPR } \\
(\%)\end{array}$ \\
\hline $\begin{array}{l}\text { Baixa } \\
\text { CAP: } 20,00 \mathrm{mg} \mathrm{L}^{-1} \\
\text { HIDRO: } 10,00 \mathrm{mg} \mathrm{L}^{-1} \\
\end{array}$ & $\begin{array}{c}19,82 \\
(99,1 \%) \\
\end{array}$ & 1,18 & $\begin{array}{c}9,95 \\
(99,5 \%) \\
\end{array}$ & 0,36 \\
\hline $\begin{array}{l}\text { Média } \\
\text { CAP: } 25,00 \mathrm{mg} \mathrm{L}^{-1} \\
\text { HIDRO: } 12,50 \mathrm{mg} \mathrm{L}^{-1}\end{array}$ & $\begin{array}{c}24,64 \\
(98,6 \%)\end{array}$ & 1,24 & $\begin{array}{c}12,32 \\
(98,6 \%)\end{array}$ & 0,27 \\
\hline $\begin{array}{l}\text { Alta } \\
\text { CAP: } 30,00 \mathrm{mg} \mathrm{L}^{-1} \\
\text { HIDRO: } 15,00 \mathrm{mg} \mathrm{L}^{-1}\end{array}$ & $\begin{array}{c}28,83 \\
(96,1 \%) \\
\end{array}$ & 0,98 & $\begin{array}{c}14,68 \\
(97,9 \%) \\
\end{array}$ & 0,86 \\
\hline \multicolumn{5}{|c|}{ EFEITO DA TEMPERATURA } \\
\hline Concentração real & \multicolumn{2}{|c|}{$25,0 \mathrm{mg} \mathrm{L}^{-1}$} & \multicolumn{2}{|c|}{$12,5 \mathrm{mg} \mathrm{L}^{-1}$} \\
\hline Temperatura & \multicolumn{4}{|c|}{$5,10,15,20,25,30,35,40,45^{\circ} \mathrm{C}$} \\
\hline Concentração média encontrada & \multicolumn{2}{|c|}{$23,47 \mathrm{mg} \mathrm{L}^{-1}$} & \multicolumn{2}{|c|}{$11,95 \mathrm{mg} \mathrm{L}^{-1}$} \\
\hline Desvio padrão relativo & \multicolumn{2}{|c|}{$1,66 \%$} & \multicolumn{2}{|c|}{$1,24 \%$} \\
\hline Erro relativo médio & \multicolumn{2}{|c|}{$6,13 \%$} & \multicolumn{2}{|c|}{$4,42 \%$} \\
\hline \multicolumn{5}{|c|}{ EFEITO DO TEMPO DE LEITURA } \\
\hline Concentração real & $25,0 \mathrm{r}$ & $\mathrm{L}^{-1}$ & $12,5 \mathrm{r}$ & $\mathrm{g} \mathrm{L}^{-1}$ \\
\hline Tempo & \multicolumn{4}{|c|}{$0,1,2,3,24,48 \mathrm{~min}$} \\
\hline Concentração média encontrada & \multicolumn{2}{|c|}{$24,42 \mathrm{mg} \mathrm{L}^{-1}$} & \multicolumn{2}{|c|}{$12,35 \mathrm{mg} \mathrm{L}^{-1}$} \\
\hline Desvio padrão relativo & \multicolumn{2}{|c|}{$1,25 \%$} & \multicolumn{2}{|c|}{$0,69 \%$} \\
\hline Erro relativo médio & \multicolumn{2}{|c|}{$2,32 \%$} & \multicolumn{2}{|c|}{$1,24 \%$} \\
\hline \multicolumn{5}{|c|}{ EFEITO DO pH } \\
\hline Concentração real & \multicolumn{2}{|c|}{$25,0 \mathrm{mg} \mathrm{L}^{-1}$} & \multicolumn{2}{|c|}{$12,5 \mathrm{mg} \mathrm{L}^{-1}$} \\
\hline $\mathrm{pH}$ & \multicolumn{4}{|c|}{$2,9-4,2-6,1$} \\
\hline Concentração média encontrada & \multicolumn{2}{|c|}{$26,25 \mathrm{mg} \mathrm{L}^{-1}$} & \multicolumn{2}{|c|}{$12,06 \mathrm{mg} \mathrm{L}^{-1}$} \\
\hline Desvio padrão relativo & \multicolumn{2}{|c|}{$6,36 \%$} & \multicolumn{2}{|c|}{$4,78 \%$} \\
\hline Erro relativo médio & \multicolumn{2}{|c|}{$3,92 \%$} & \multicolumn{2}{|c|}{4,37} \\
\hline
\end{tabular}

fazendo com que os desvios observados sejam superiores ao limite de 5\% imposto pela legislação para estudos de precisão. Em função desta constatação, recomenda-se que os modelos sejam desenvolvidos em valores de $\mathrm{pH}$ próximos daqueles apresentados pelas amostras que se deseja analisar.

Análise de medicamentos e ensaios de dissolução

Os resultados obtidos na análise das misturas sintéticas do conjunto de validação externa e das amostras de medicamentos são apresentados na Tabela 2, incluindo-se os resultados obtidos por aplicação da técnica cromatográfica padrão. Se for considerado que o resultado obtido pela aplicação da técnica cromatográfica padrão corresponde ao valor verdadeiro, podemos observar, em primeira instância, a existência de inconsistências ente os valores reais e declarados na bula dos medicamentos, principalmente para as amostras de medicamento de referência. A referida diferença, entretanto, encontra-se no limite dos valores de referência preconizados pela ANVISA (até 10\%). Comparando-se os resultados proporcionados pela técnica cromatográfica padrão e pelo método espectroscópico multivariado proposto, é possível observar uma boa aproximação na análise dos medicamentos de referência e genérico. Em ambos os casos, os erros de previsão apresentados pelo método multivariado são da ordem de $6 \%$ para captopril e entre 2 e $9 \%$ para hidroclorotiazida. Para o medicamento similar observam-se resultados discrepantes, com erros da ordem de $15 \%$, provavelmente em função da presença de excipientes que modificam o perfil espectral na região monitorada.

De acordo com os perfis de dissolução obtidos por análise cromatográfica do medicamento de referência (Figura 3), a dissolução do captopril é bastante rápida, o que equivale a aproximadamente $100 \%$ do valor informado na bula a partir de 15 min de ensaio. Por

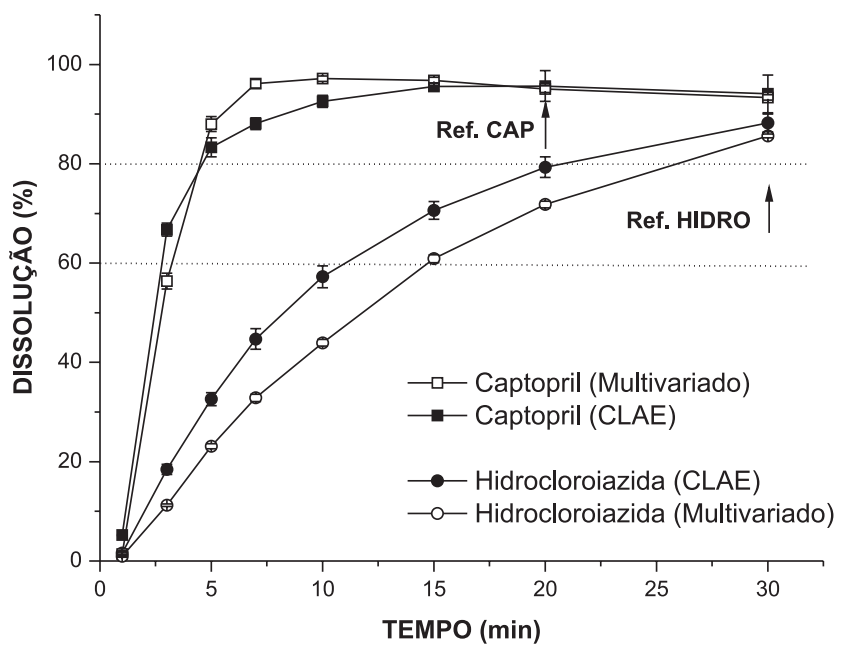

Figura 3. Perfil de dissolução do medicamento de referência (captoprilhidroclorotiazida) obtido por método cromatográfico de referência e método espectroscópico multivariado

Tabela 2. Erros relativos médios obtidos na determinação de captopril e hidroclorotiazida em misturas sintéticas (validação externa) e de medicamentos utilizando-se modelo multivariado (espectros centrados na média e duas VL, $\mathrm{n}=3$ )

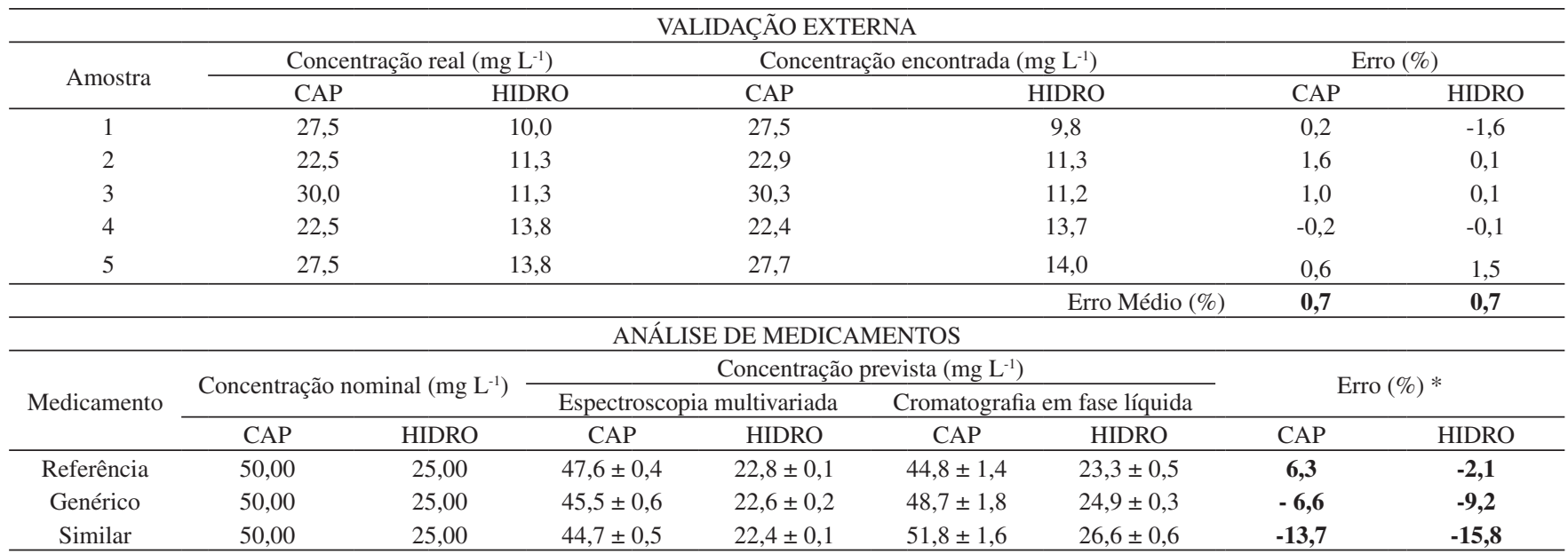

* Erro relativo em relação ao valor determinado por técnica cromatográfica padrão. 
sua vez, a hidroclorotiazida dissolve mais lentamente, alcançando valores da ordem de $85 \%$ do valor nominal em 30 min de ensaio. Estes resultados são coerentes com os limites impostos pela Farmacopeia Americana, ${ }^{14}$ os quais correspondem à dissolução de pelo menos $80 \%$ do teor nominal de captopril e $60 \%$ do teor nominal de hidroclorotiazida, em tempos de 20 e $30 \mathrm{~min}$, respectivamente. Os perfis de dissolução obtidos por espectroscopia multivariada são bastante similares aos obtidos pelo método cromatográfico padrão (Figura 3), com discrepâncias que podem ser devidas a imprecisões na coleta de amostras, o que, em função da rápida dissolução dos medicamentos, torna-se crítico nos primeiros momentos do ensaio.

Resultados igualmente coerentes foram observados em ensaios de dissolução envolvendo os medicamentos genérico e similar (Figura $1 \mathrm{~S}$, material suplementar).

\section{Associação zidovudina-lamivudina}

Zidovudina e lamivudina apresentam forte absorção na região ultravioleta, absorção esta que é caracterizada por dois máximos bem definidos (Figura 4). A interferência espectral entre estas espécies é bastante evidente, o que, em primeira análise, sugere inconveniência de sistemas convencionais de calibração. Trata-se de um argumento relevante, uma vez que justifica o estudo de sistemas alternativos de calibração.

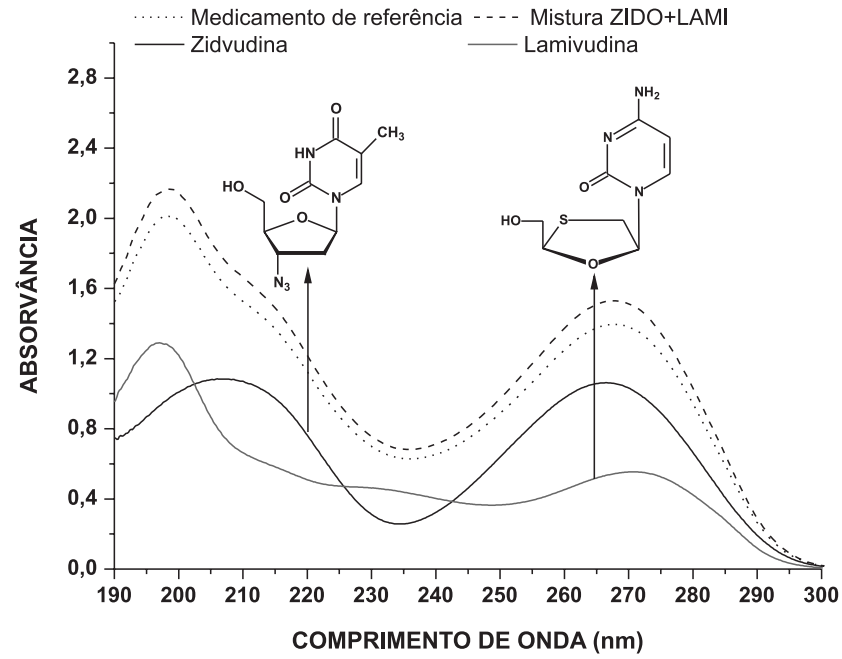

Figura 4. Espectro eletrônico de soluções aquosas de zidovudina $(24,0 \mathrm{mg}$ $\left.\mathrm{L}^{-1}\right)$, lamivudina $\left(12,0 \mathrm{mg} \mathrm{L}^{-1}\right)$, mistura sintética e medicamento de referência

Como no caso anterior, modelos multivariados foram desenvolvidos com diversos tipos de pré-processamento de sinais e diferentes números de VL. Os resultados, apresentados na forma de erro médio na etapa de validação externa (Figura 5), indicam que todos os modelos desenvolvidos apresentam capacidade de previsão comparável, fornecendo erros médios inferiores a 2,5\% (zidovudina) e 1,5\% (lamivudina). Para aplicação em análises subsequentes foi selecionado o modelo elaborado com espectros centrados na média (pré-processamento 1) e três variáveis latentes, as quais são responsáveis por 99,79\% da variância dos dados de concentração e 99,96\% da variância dos dados espectrais.

\section{Validação do método multivariado}

Como no caso anterior, a precisão foi avaliada em dois níveis diferentes (repetibilidade e reprodutibilidade), por meio de análise em triplicata de três misturas sintéticas contendo concentrações altas, médias e baixas de ambos os fármacos. Os resultados desta avaliação (Tabela 3) demonstram uma excelente precisão da metodologia

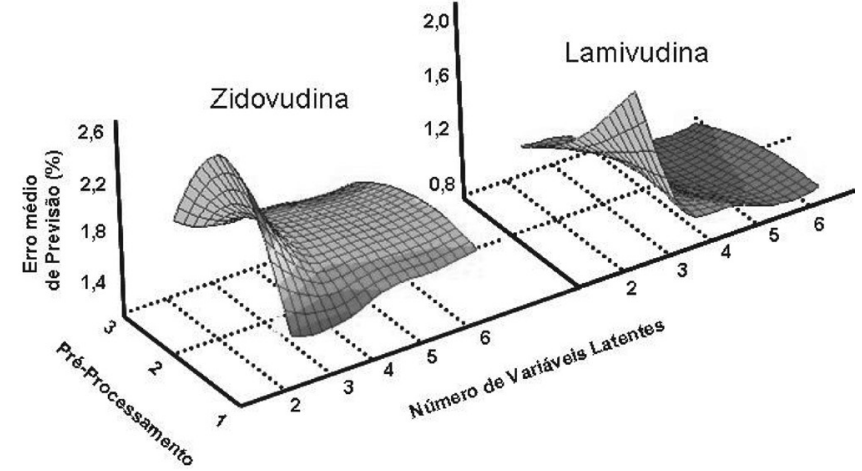

Figura 5. Evolução do erro médio de previsão (zidovudina-lamivudina) na fase de validação externa em função do número de variáveis latentes e do tipo de pré-processamento (1: centrado na média, 2: autoescalado, 3: alisado e derivado)

analítica em avaliação, com desvios padrão relativos entre $0,10 \mathrm{e}$ $0,64 \%$, portanto, dentro dos limites estabelecidos pela ANVISA (5\%).

Adicionalmente, os resultados apresentados na Tabela 3 permitem avaliar a exatidão da metodologia proposta, em função da recuperação das concentrações utilizadas no ensaio. Neste caso, a

Tabela 3. Resultados da determinação de lamivudina e zidovudina em misturas sintéticas utilizadas para avaliação da reprodutibilidade $(n=3)$ e robustez

\begin{tabular}{|c|c|c|c|c|}
\hline \multirow[b]{2}{*}{ Concentração } & \multicolumn{2}{|c|}{ Zidovudina } & \multicolumn{2}{|c|}{ Lamivudina } \\
\hline & $\begin{array}{c}\text { Média } \\
\left(\mathrm{mg} \mathrm{L}^{-1}\right)\end{array}$ & $\begin{array}{c}\text { DPR } \\
(\%)\end{array}$ & $\begin{array}{c}\text { Média } \\
\left(\mathrm{mg} \mathrm{L}^{-1}\right)\end{array}$ & $\begin{array}{l}\text { DPR } \\
(\%)\end{array}$ \\
\hline $\begin{array}{l}\text { Baixa } \\
\text { ZIDO: } 19,00 \mathrm{mg} \mathrm{L}^{-1} \\
\text { LAMI: } 10,00 \mathrm{mg} \mathrm{L}^{-1}\end{array}$ & $\begin{array}{c}19,79 \\
(104,1 \%)\end{array}$ & 0,28 & $\begin{array}{c}9,93 \\
(99,3 \%) \\
\end{array}$ & 0,20 \\
\hline $\begin{array}{l}\text { Média } \\
\text { ZIDO: } 24,00 \mathrm{mg} \mathrm{L}^{-1} \\
\text { LAMI: } 12,00 \mathrm{mg} \mathrm{L}^{-1} \\
\end{array}$ & $\begin{array}{c}24,43 \\
(101,8 \%) \\
\end{array}$ & 0,64 & $\begin{array}{c}11,64 \\
(97,0 \%) \\
\end{array}$ & 0,39 \\
\hline $\begin{array}{l}\text { Alta } \\
\text { ZIDO: } 29,00 \mathrm{mg} \mathrm{L}^{-1} \\
\text { LAMI: } 14,00 \mathrm{mg} \mathrm{L}^{-1} \\
\end{array}$ & $\begin{array}{c}29,11 \\
(100,4 \%) \\
\end{array}$ & 0,33 & $\begin{array}{c}13,69 \\
(97,8 \%) \\
\end{array}$ & 0,10 \\
\hline \multicolumn{5}{|c|}{ EFEITO DA TEMPERATURA } \\
\hline Concentração real & \multicolumn{2}{|c|}{$24,0 \mathrm{mg} \mathrm{L}^{-1}$} & \multicolumn{2}{|c|}{$12,0 \mathrm{mg} \mathrm{L}^{-1}$} \\
\hline Temperatura & \multicolumn{4}{|c|}{$5,10,15,20,25,30,35,40,45^{\circ} \mathrm{C}$} \\
\hline Concentração média encontrada & \multicolumn{2}{|c|}{$23,17 \mathrm{mg} \mathrm{L}^{-1}$} & \multicolumn{2}{|c|}{$11,98 \mathrm{mg} \mathrm{L}^{-1}$} \\
\hline Desvio padrão relativo & \multicolumn{2}{|c|}{$1,54 \%$} & \multicolumn{2}{|c|}{$2,77 \%$} \\
\hline Erro relativo médio & \multicolumn{2}{|c|}{$3,46 \%$} & \multicolumn{2}{|c|}{$0,17 \%$} \\
\hline \multicolumn{5}{|c|}{ EFEITO DO TEMPO DE LEITURA } \\
\hline Concentração real & \multicolumn{2}{|c|}{$24,0 \mathrm{mg} \mathrm{L}^{-1}$} & \multicolumn{2}{|c|}{$12,0 \mathrm{mg} \mathrm{L}^{-1}$} \\
\hline Tempo & \multicolumn{4}{|c|}{$0,1,2,3,24,48 \mathrm{~min}$} \\
\hline Concentração média encontrada & \multicolumn{2}{|c|}{$23,40 \mathrm{mg} \mathrm{L}^{-1}$} & \multicolumn{2}{|c|}{$11,71 \mathrm{mg} \mathrm{L}^{-1}$} \\
\hline Desvio padrão relativo & \multicolumn{2}{|c|}{$0,46 \%$} & \multicolumn{2}{|c|}{$0,27 \%$} \\
\hline Erro relativo médio & \multicolumn{2}{|c|}{$2,49 \%$} & \multicolumn{2}{|c|}{$2,42 \%$} \\
\hline \multicolumn{5}{|c|}{ EFEITO DO pH } \\
\hline \multirow[t]{2}{*}{ Concentração real } & \multicolumn{2}{|c|}{$24,0 \mathrm{mg} \mathrm{L}^{-1}$} & \multicolumn{2}{|c|}{$12,0 \mathrm{mg} \mathrm{L}^{-1}$} \\
\hline & $\begin{array}{l}\text { Conc. } \\
\text { prevista } \\
\left(\mathrm{mg} \mathrm{L}^{-1}\right)\end{array}$ & Erro $(\%)$ & $\begin{array}{l}\text { Conc. } \\
\text { prevista } \\
\left(\mathrm{mg} \mathrm{L}^{-1}\right)\end{array}$ & Erro $(\%)$ \\
\hline pH 3 & 32,23 & $(34,29)$ & 7,31 & $(-39,09)$ \\
\hline pH 5 & 24,72 & $(2,99)$ & 11,33 & $(-5,62)$ \\
\hline pH 6 & 24,15 & $(0,62)$ & 12,11 & $(0,93)$ \\
\hline $\mathrm{pH} 7$ & 22,36 & $(-6,82)$ & 11,28 & $(-5,98)$ \\
\hline pH 9 & 21,28 & $(-11,35)$ & 10,94 & $(-8,81)$ \\
\hline $\mathrm{pH} 10$ & 23,07 & $(-3,86)$ & 7,90 & $(-34,13)$ \\
\hline
\end{tabular}


recuperação alcança valores entre 97,0 e $104,1 \%$, o que poder ser considerado satisfatório.

A robustez do método foi avaliada através da análise de uma mistura sintética contendo $24,0 \mathrm{mg} \mathrm{L}^{-1}$ de AZT e $12,0 \mathrm{mg} \mathrm{L}^{-1}$ de $3 \mathrm{TC}$ em condições de diferentes valores de $\mathrm{pH}$, temperatura e tempo de leitura. Os resultados (Tabela 3) indicam que mudanças na temperatura ou no tempo de leitura não provocam modificações significativas do sinal espectral, e que, consequentemente, não prejudicam a capacidade de previsão do modelo. Neste estudo, desvios médios inferiores a $3 \%$ e erros de previsão médios inferiores a $4 \%$ foram obtidos, valores que se encontram dentro dos limites estabelecidos para este tipo de método.

Diferentemente, mudanças no $\mathrm{pH}$ das amostras levam à obtenção de elevados erros de previsão, principalmente em razão das significativas mudanças provocadas no perfil espectral dos substratos. Zidovudina e lamivudina apresentam valores de pKa de 9,8 e 4,3, respectivamente, o que faz com que o equilíbrio de protonação seja sensivelmente modificado em valores de $\mathrm{pH}$ próximos a estes valores. Desta forma, a capacidade de previsão do modelo é seriamente comprometida em meio ácido ( $\mathrm{pH} 3$ ) ou básico ( $\mathrm{pH} 10)$, o que leva à obtenção de erros de previsão da ordem de $30 \%$. Trabalhando-se em $\mathrm{pH}$ entre 5 e 7, uma boa capacidade de previsão é observada (erros de previsão inferiores a 7\%), enquanto que se trabalhando no mesmo $\mathrm{pH}$ em que o modelo foi desenvolvido ( $\mathrm{pH}$ 6), os erro de previsão são significativamente reduzidos $(<1 \%)$. Estas observações salientam a necessidade de um controle de $\mathrm{pH}$ utilizando-se, por exemplo, soluções tampão.

Análise de medicamentos e ensaios de dissolução

Utilizando-se o modelo de melhor desempenho, foram observados erros médios de previsão da ordem de $1 \%$ para ambos os fármacos nas amostras de validação externa (Tabela 4). Na análise de medicamentos (Tabela 4), erros de previsão médios inferiores a $8 \%$ foram observados, em relação aos valores medidos pela técnica cromatográfica padrão, o que atesta a boa capacidade do sistema multivariado para quantificação de associações de lamivudina e zidovudina.

O perfil de dissolução do fármaco de referência, avaliado por cromatografia em fase líquida, é apresentado na Figura 6. Em função deste comportamento, o medicamento pode ser considerado de pronta liberação, alcançando aproximadamente $80 \%$ da sua concentração nominal nos primeiros $5 \mathrm{~min}$ de teste. Este resultado está em conformidade com a norma brasileira, ${ }^{15}$ que estabelece uma dissolução não menor que $80 \%$ em 60 min de ensaio. Os perfis de dissolução obtidos pelo método espectroscópico multivariado são bastante similares, com discrepâncias provavelmente decorrentes da imprecisão associada à rápida dissolução dos medicamentos.

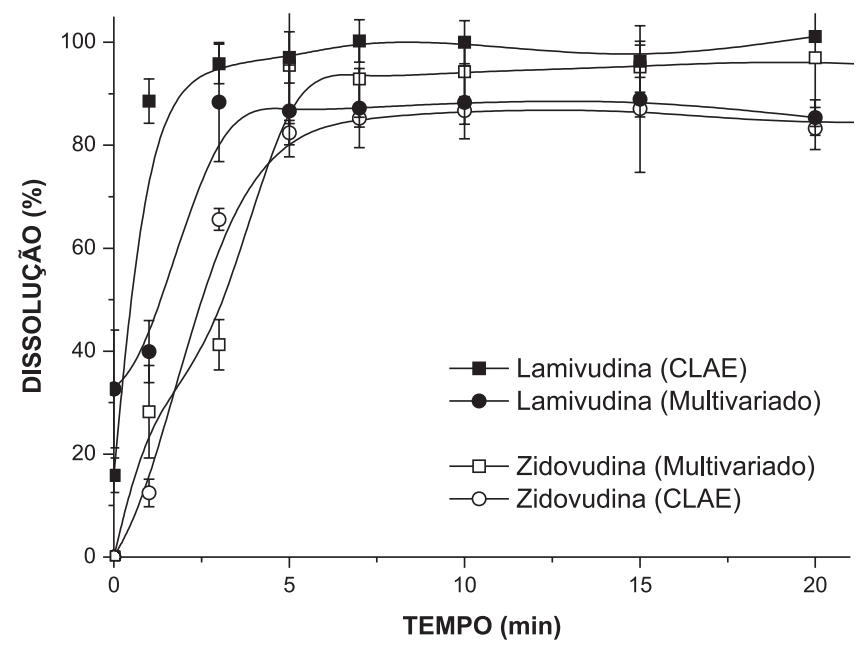

Figura 6. Perfil de dissolução do medicamento de referência (zidovudinalamivudina) obtido por método cromatográfico de referência e método espectroscópico multivariado

Resultados igualmente coerentes foram observados em ensaios de dissolução envolvendo medicamentos genéricos (Figura 2S, material suplementar).

\section{CONCLUSÕES}

A regressão por mínimos quadrados parciais (PLSR) permite o desenvolvimento de modelos espectroscópicos multivariados de excelente capacidade de previsão, o que permite a determinação simultânea de fármacos em associações farmacêuticas (captoprilhidroclorotiazida e zidovudina-lamivudina), mesmo na presença de importante interferência espectral. Os modelos referidos podem ser utilizados com sucesso na quantificação dos fármacos em medicamentos e em ensaios de dissolução, com excelente aproximação em relação aos resultados proporcionados pelas técnicas cromatográficas padrão. Trata-se de uma constatação extremamente relevante, principalmente em função da usual complexidade das rotinas analíticas fundamentadas em cromatografia em fase líquida e da simplicidade das rotinas multivariadas fundamentadas em espectroscopia UV-VIS.

Tabela 4. Erros relativos médios obtidos na determinação de zidovudina e lamivudina em misturas sintéticas (validação externa) e de medicamentos utilizandose modelo multivariado (espectros centrados na média e três VL, n=3)

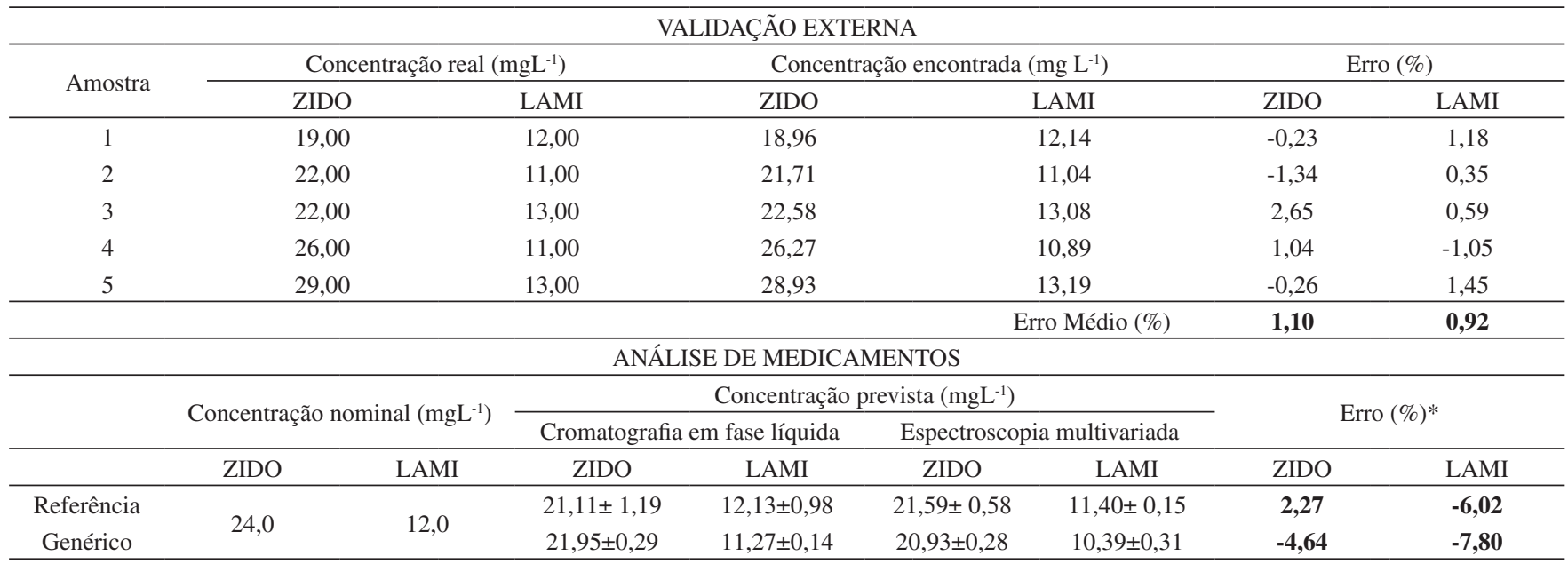

* Erro relativo em relação ao valor determinado por técnica cromatográfica padrão. 


\section{MATERIAL SUPLEMENTAR}

Está disponível em http://quimicanova.sbq.org.br, na forma de arquivo pdf, com acesso livre. Na Figura 1S apresenta-se o perfil de dissolução do medicamento genérico da associação captoprilhidroclorotiazida, obtido por método cromatográfico de referência e método espectroscópico multivariado. Na Figura $2 \mathrm{~S}$ apresenta-se o perfil de dissolução do medicamento genérico da associação zidovudina-lamivudina, obtido por método cromatográfico de referência e método espectroscópico multivariado.

\section{REFERÊNCIAS}

1. Chowdary, K. P. R.; Rajyalakshmy, Y.; East Pharm. 1987, 30, 51.

2. Dalmora, S. L.; Nogueira, D. R.; Calegari, G. Z.; Bergamo, A. C.; Stamm, F. P.; Quim. Nova 2010, 33, 1150.

3. Soares, M. F. L. R.; Soares Sobrinho, J. L.; Grangeiro Junior, S.; Silva, K. E. R.; Alves, L. D. S.; Rolim Neto, P. J.; Quim. Nova 2010, 33, 478

4. Bonfilio, R.; Mendonça, T. F.; Pereira, G. R.; Araújo, M. B.; Tarley, C. R. T.; Quim. Nova 2010, 33, 377.

5. Wrasse-Sangoi, M.; Secretti, L. T.; Diefenbach, I. F.; Rolim, C. M. B.; Sangoi, M. S.; Quim. Nova 2010, 33, 1330.
6. Barboza, F. M.; Vecchia, D. D.; Pereira, A. V.; Stulzer, H. K.; Silva, M. A. S.; Quim. Nova 2010, 33, 747.

7. Markopoulou, C. K.; Malliou, E. T.; Koundourellis, J. E.; II Farmaco 2005, 60, 755

8. Markopoulou, C. K.; Malliou, E. T.; Koundourellis, J. E.; J. Pharm. Biomed. Anal. 2005, 36, 249.

9. Escandar, G. M.; Damiani, P. C.; Goicoechea, H. C.; Olivieri, A. C.; Microchem. J. 2006, 82, 29.

10. Rossignoli, P.; Pontarolo, R.; Correr, C. J.; Cordeiro, G. A.; PeraltaZamora, P.; Quim. Nova 2008, 31, 1285.

11. Barthus, R. C.; Mazo, L. H.; Poppi, R. J.; Quim. Nova 2007, 30, 1638.

12. Sena, M. M.; Freitas, C .B.; Silva, L. C.; Pérez, C. N.; de Paula, Y. O.; Quim. Nova 2007, 30, 75.

13. Dinç, E.; Serin, C.; Tuğcu-Demiröz,F.; Doğanay, T.; Int. J. Pharm. 2003, 250,339 .

14. U.S. Pharmacopeial Convention; The United States Parmacopeia. USP 30; NF 25, Rockville, 2007, p. 244 e 3492.

15. ANVISA - Agência Nacional de Vigilância Sanitária; Resolução$R D C N^{o}$ 31, de 31/8/ 2010. Dispõe sobre a realização dos Estudos de Equivalência Farmacêutica e de Perfil de Dissolução Comparativo, disponível em http://bvsms.saude.gov.br/bvs/saudelegis/anvisa/2010/ res0031_11_08_2010.html, acessada em Outubro 2010. 


\section{AVALIAÇÃO DO PERFIL DE DISSOLUÇÃO DE MEDICAMENTOS UTILIZANDO-SE ESPECTROSCOPIA ELETRÔNICA MULTIVARIADA}

Juliana dos Santos, Gilcélia A. Cordeiro, Noemi Nagata e Patricio Peralta-Zamora*

Departamento de Química, Universidade Federal do Paraná, CP 19081, 81531-990 Curitiba - PR, Brasil

Letícia Norma Carpentieri Rodrigues

Departamento de Ciências Exatas e da Terra, Universidade Federal de São Paulo, 09972-270 Diadema - SP, Brasil

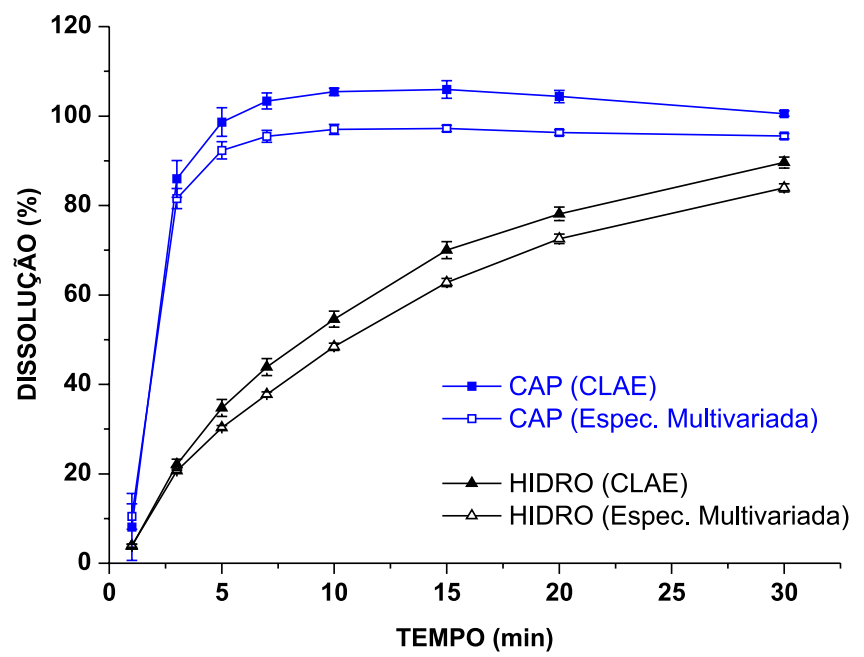

Figura 1S. Perfil de dissolução do medicamento genérico (captoprilhidroclorotiazida) obtido por método cromatográfico de referência e método espectroscópico multivariado

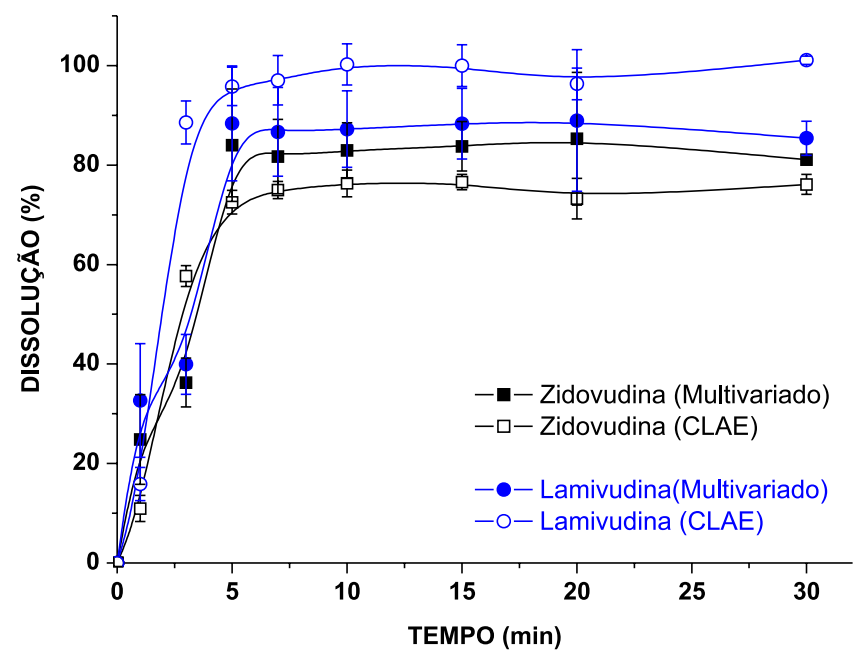

Figura 2S. Perfil de dissolução do medicamento genérico (zidovudinalamivudina) obtido por método cromatográfico de referência e método espectroscópico multivariado 